\title{
IMPACT OF ANTENNA CHOICES ON THE RELIABILITY OF MOBILE BROADBAND TRANSMISSION AT MILLIMETRE-WAVE FREQUENCIES
}

\author{
António Gusmão, Rui Dinis and Paulo Silva \\ CAPS, Instituto Superior Técnico, Av. Rovisco Pais, \\ 1096 Lisboa Codex, Portugal
}

\begin{abstract}
This paper deals with the impact of several antenna choices on the radio transmission performance within a cellular Mobile Broadband System (MBS) currently under research in Europe. Several antenna types are considered, namely switchable-beam antennas and adaptive antennas employing a phased array approach. Several simulation results are presented and discussed: they show that some directivity in the MS antenna is recommendable for an acceptable performance, and that the proposed adaptive MS antennas can be of interest for MBS, namely for advanced system implementation stages.
\end{abstract}

\section{INTRODUCTION}

The implementation of a cellular system, able to offer to mobile users an ATM-based radio access to the future IBCN, certainly represents a considerable challenge: the range of services is very wide (multimedia, HDTV contribution, etc.), with a variety of requirements and characteristics, including service bit rates of several tens of Mbit/s, much higher than in current mobile radio systems; it is necessary to resort to mm-wave frequencies for radio transmission, since the required high gross bit rates imply a very wide band, which is not available below $30 \mathrm{GHz}$.

Within the European RACE Project R2067-MBS, a set of preliminary specifications has been proposed for the air interface of a cellular Mobile $\underline{B}$ roadband $\underline{S} y s t e m(\mathrm{MBS})^{1}$, namely the following:

- A 4-OQAM scheme (2 bits per symbol), allowing the use of strongly nonlinear power amplifiers, in a first system implementation stage; also a compatible 16-OQAM scheme (4 bits per symbol) in later implementation stages.

- Two gross symbol rates: $40 \mathrm{Msymbol} / \mathrm{s}$, for selected indoor environments (small areas); 20 Msymbol/s, for other indoor environments (large areas) and for outdoor environments.

- A flexible TDMA/FDMA scheme, allowing the use of multiple slots on a single carrier and several carriers (up to four) in parallel, when the highest user bit rates are intended. 
The main problems to solve in order to implement such a cellular system, at reasonable costs, are related to radio propagation conditions in outdoor environments. The activities carried out within the MBS/RACE Project with regards to the characterization of the radio channels have clearly shown that this characterization strongly depends on cellular configuration and antenna choices; appropriate antenna/cell design options certainly are one of the key issues for the RF feasibility of MBS.

Having in mind the MBS requirements, a class of adaptive, low-cost, "serial OQAMtype" receivers was proposed in ref. 2 , with decision-feedback equalization (DFE) and three alternatives with regards to the use of space diversity: no diversity at all; conventional, RSSIdriven, switched diversity; two-branch combining diversity that can be regarded as a wideband version of the well-known "maximal-ratio combining" (MRC).

In this paper, we study the impact of the antenna choices on the performance achieved through the use of the adaptive receivers reported above, within appropriate cell structures (designed to cope with the hostile propagation conditions, allowing a reliable transmission).

In Sec. 2, we report some of the MBS/RACE results on transmission performance, which illustrate the antenna/receiver tradeoff problem. Sec. 3 presents guidelines for the design of adaptive antennas suitable for MBS. A set of simulation results is shown and discussed in Sec. 4. Some final remarks, in Sec. 5, conclude the paper.

\section{TRANSMISSION PERFORMANCE AND THE ANTENNA/RECEIVER TRADEOFF PROBLEM}

An extensive simulation work carried out within the MBS/RACE project ${ }^{3}$, as well as some experimental work using the MBS/RACE demonstrator' ${ }^{\prime}$, have shown that the radio propagation conditions can give rise to both "delay spread difficulties" (heavy distortion effects on the required wideband signals) and "coverage difficulties" (obstruction of the LOS path whenever relevant reflections are not available). A certain directivity (in both the horizontal and vertical planes) of BS and MS antennas was shown to help in avoiding too a high delay spread in certain scenarios, therefore ensuring a good performance when equalization schemes of moderate complexity are employed (moreover, antenna directivity can also allow reduced transmit power requirements and an increased frequency reuse). By adopting circular polarization, a delay spread reduction was also observed.

A class of adaptive receivers previously proposed by the authors ${ }^{2}$ has been assumed for

the above-mentioned performance evaluation. As in GSM receivers, channel estimation/equalization and off-line processing, on a burst-by-burst basis, are employed in these receivers. During the training mode of the adaptive operation, prior to message detection, the estimates of one (no diversity and switched diversity) or two (approximate "maximal ratio" combining diversity) overall Channel Impulse Responses (CIR) are obtained through a standard correlation technique, thanks to a 30-bit long training sequence, located in the middle of the burst. The CIR estimates are then used, on the one hand, to obtain the tap coefficients of one (no diversity and switched diversity) or two (combining diversity), approximately matched, FIR filters; on the other hand, they are used to calculate the tap coefficients of the single DFE required, under an MMSE (Minimum Mean Square Error) criterium. An additional tracking mode (employing an LMS iterative algorithm) allows the receiver to follow and compensate for the channel changes from the midamble to both burst edges.

Performances were evaluated in ref. 3 under the following antenna assumptions, for a selected outdoor scenario (consisting of a $30 \mathrm{~m}$ wide city street where a "typical" bus can give rise to strong back-reflected rays): either omnidirectional or switchable-beam antennas, with 9 sectors and sector selection on an average power basis, were considered in the mobile station 